\title{
The relevance of the Spanish Society for Medical Oncology (SEOM)'s clinical oncology guidelines
}

\author{
Dolores Isla Casado • Antonio González-Martín · Emilio Alba Conejo
}

$\mathrm{I}$ this issue of Clinical and Translational Oncology, the Spanish Society for Medical Oncology (SEOM) publishes a group of the Clinical Guidelines that are the result of an ambitious educational programme. The main objective of this programme was to periodically create and actualise a set of clinical guidelines mainly focused on the medical treatment for the majority of solid tumours and cancerrelated or therapy-related symptoms.

Clinical guidelines have increasingly become a common part of clinical practice during the past decade. They are defined by the Institute of Medicine as "systematically developed statements to assist practitioner and patient decisions about appropriate health care for specific clinical circumstances" [1]. Appropriate care involves assessing the balance between the sum of the benefits and the sum of the risks [2].

There are several factors that have stimulated increased interest in the development of clinical guidelines. Among the most relevant reasons are the exponential increment of medical publications, rising healthcare costs, the use of more expensive technologies, variations in service delivery among providers even within the same city or geographic region and the aspiration of healthcare physicians to offer

D. Isla Casado

SEOM Clinical Guidelines Coordinator

Servicio de Oncología Médica

Hospital Clínico Universitario Lozano Blesa

Zaragoza, Spain

A. González-Martín (西)

Senior Editor for Medical Oncology

Servicio de Oncología Médica

Centro Oncológico MD Anderson International España

C/ Arturo Soria, 270

ES-28033 Madrid, Spain

e-mail: agonzalezm@seom.org

E. Alba Conejo

Chairman of SEOM

Servicio de Oncología Médica

Hospital Clínico Universitario Virgen de la Victoria

Málaga, Spain the best possible care to their cancer patients. In this scenario, clinical guidelines are considered as a tool for giving the patients the best care based on the most consistent scientific evidence. Therapy based on guidelines has been shown to lead to better clinical outcomes compared with locally adapted therapeutic strategies and this would also lead to improved quality of care for the patients.

Several objectives can be assigned to clinical guidelines. The main aim is usually to improve the quality of the medical care. However, other important objectives to consider are to facilitate the decision-making process for the treating physician, to promote efficient use of the health system resources and to set the basis for performing an evaluation of the quality of medical care. Clinical guidelines' highest quality probably lies in the methodology for constructing them based on the comprehensive review of the literature and the most convincing evidence-based medicine available.

Nowadays, there are many clinical guidelines in oncology, produced by different institutions, governments, scientific societies and cooperative groups. So why has SEOM launched a new group of clinical guidelines? This initiative is the result of the intent to adapt the best evidence-based knowledge in medical oncology to the specific scenario of the Spanish health care system and to actively cooperate with the health authorities in the homogenisation of medical oncology care among the different communities in Spain. For this purpose, the board of SEOM created a working group in 2008 with the aim of coordinating this project.

The clinical guidelines working group established the methodology and the basis for building each guideline. They defined some common characteristics for each guideline with special emphasis on being clear and concise but complete at the same time, and the use of tables or algorithms for facilitating comprehension. It should be highlighted that the recommendations made by the clinical guidelines should be in accordance with the approvals obtained by the different therapies in Spain at the moment of writing the guideline. Regarding the authors, a first panel of experts was nominated in order to write the first draft of the guideline. Then, a second panel of experts also nomi- 
nated by SEOM reviewed the draft and proposed a definitive document to be approved. Finally, the board of SEOM and the Clinical Guidelines working group reviewed the document and gave the final approval before the release.

The guidelines have covered the medical oncologic treatment of the most relevant and frequent malignancies, and also the supportive care for the main disease-related or therapy-related symptoms. Each guideline has been well structured and includes the following parts: introduction, staging, diagnosis, therapy and follow-up. Again, the inclusion of algorithms to clarify the recommendations was specifically encouraged. For the supportive care guidelines it was not possible to establish such a well defined structure fitting all the subjects. Finally, the guidelines will be updated annually in order to maintain their validity and introduce the most recent advances in medical oncology.

In 2009, some guidelines were periodically published in the Yellow Series of Clinical and Translational Oncology $[4,5]$. However, as an important number of guidelines were ready to be delivered, it was decided by the Editorial
Board of Clinical and Translational Oncology to publish them all together in this ordinary issue in order to keep the information as up-to-date as possible. The reader will find in this issue the guidelines for the treatment of early breast cancer [6], metastatic breast cancer [7], adjuvant treatment of colorectal cancer [8], advanced colorectal cancer [9], non-small-cell lung cancer (an update) [10], head and neck cancer [11], nasopharyngeal carcinoma [12], Hodgkin's lymphoma [13], non-Hodgkin's lymphoma [14], diffuse large B-cell lymphoma [15] and antiemetic prophylaxis for patients receiving chemotherapy [16]. Finally, it is scheduled to publish the remaining guidelines, which are under revision at the moment, in the first trimester of 2011.

The SEOM Guidelines working group and the members of the SEOM board believe that these guidelines are a valuable tool not only for the medical oncologist in our country but also for other physicians implicated in cancer patient care. We hope that the concerted efforts of the authors, reviewers and coordinators will translate into better care for cancer patients.

\section{References}

1. Field MJ, Lhr KN (eds) (1990) Clinical practice guidelines: direction for a new program. Institute of Medicine, Committee on Clinical Practice Guidelines, Washington, DC. National Academy Press

2. Park EP, Fink A, Brook RH et al (1986) Physician ratings of appropriate indications for six medical and surgical procedures. Am J Public Health 76: 766-772

3. Woolf S, Grol R, Hutchinson A et al (1999) Potential benefits, limitations, and harms of clinical guidelines. Br Med J 318:527-530

4. Felip E, Garrido P, Trigo JM et al (2009) SEOM guidelines for the management of non-small cell lung cancer (NSCLC). Clin Transl Oncol 11:284289

5. Artal A, Dómine M, Font A et al (2010) SEOM clinical guidelines for the treatment of small-cell lung cancer. Clin Transl Oncol 12:27-31

6. del Barco Berrón S, Ciruelos Gil E, Tusquets Trías de Bes I et al (2010) SEOM clinical guidelines for the treatment of early breast cancer. Clin Transl Oncol 12:711-718

7. Álvarez López I, de la Haba Rodríguez J, Ruiz Simón A et al (2010) SEOM clinical guidelines for the treatment of metastatic breast cancer. Clin Transl Oncol 12:719-723

8. Grávalos Castro C, Maurel Santasusana J, Rivera Herrero F et al (2010) SEOM clinical guidelines for the adjuvant treatment of colorectal cancer. Clin Transl Oncol 12:724-728

9. García-Carbonero R, Gómez España MA, Casado Sáenz E et al (2010) SEOM clinical guidelines for the treatment of advanced colorectal cancer. Clin Transl Oncol 12:729-734

10. Trigo Pérez JM, Garrido López P, Felip Font E, Isla Casado D (2010) SEOM clinical guidelines for the treatment of non-small-cell lung cancer: an updated edition. Clin Transl Oncol 12:735-741

11. Mesía Nin R, Pastor Borgoñón M, Cruz Hernández JJ, Isla Casado D (2010) SEOM clinical guidelines for the treatment of head and neck cancer. Clin Transl Oncol 12:742-748
12. Pastor Borgoñón M, Mesía Nin R, Cruz Hernández JJ, Isla Casado D (2010) SEOM clinical guidelines for the treatment of nasopharyngeal carcinoma. Clin Transl Oncol 12:749-752

13. Quero Blanco C, García Arroyo R, Provencio Pulla $M$ et al (2010) SEOM clinical guidelines for the treatment of Hodgkin's lymphoma. Clin Transl Oncol 12:753-759

14. Rodríguez-Abreu D, Llanos Muñoz M, Provencio Pulla M et al (2010) SEOM clinical guidelines for the treatment of follicular non-Hodgkin's lymphoma. Clin Transl Oncol 12:760-764

15. Gómez Codina J, Sabín Domínguez P, Provencio Pulla M et al (2010) SEOM clinical guidelines for the treatment of diffuse large B-cell lymphoma. Clin Transl Oncol 12:765-769

16. García Gómez J, Pérez López ME, García Mata J, Isla Casado D (2010) SEOM clinical guidelines for the treatment of antiemetic prophylaxis in cancer patients receiving chemotherapy. Clin Transl Oncol 12:770-774 